http://jmscr.igmpublication.org/home/ ISSN (e)-2347-176x ISSN (p) 2455-0450

crossref DOI: https://dx.doi.org/10.18535/jmscr/v7i12.87

\title{
Management of Isolated Orbital Floor Fracture with Prolene and Titanium Mesh - A Comparative Study
}

Authors

\section{Dr C Edwin Emperor ${ }^{1}$, Dr K Adhil Ahmed Yameen², Dr P Suresh Kumar Dr T Chenthil Sivamuthu ${ }^{4}$, Dr M Basker Kanagaraj ${ }^{5}$}

${ }^{1,4}$ Associate Professor of Plastic Surgery, Tirunelveli Medical College, Tirunelveli, Tamilnadu, India

${ }^{2,5}$ Assistant Professor of Plastic Surgery, Tirunelveli Medical College, Tirunelveli, Tamilnadu, India

${ }^{3}$ Professor and HOD of Plastic Surgery, Tirunelveli Medical College, Tirunelveli, Tamilnadu, India

\section{Abstract}

Orbital fractures are one of the most frequent consequences following midfacial trauma. If not treated they can lead to serious optical complications such as; double vision (diplopia), restriction of ocular motility, enophthalmos. Autogenic bone graft although still wide and effectively used for reconstruction of fractured orbital wall present some disadvantages such as morbidity of donor site, resorption, time consuming procedure and long post-operative care. Because of that introduction of new materials for orbital reconstruction seems to be desirable.

\section{Introduction}

The term orbital blowout fracture was first coined by Smith \& Regan in 1957 and categorized the pure form where the orbital floor is exclusively involved and the impure form of the injury when there was a concomitant fracture of the orbital rim. The pathogenesis remains contentious as three distinct mechanisms of injury have been proposed: Increased intra-orbital Hydraulic pressure, the buckling force theory and the hypothesis of direct globe-to-wall contact.

Prevalence of pure orbital blowout fractures ranged from $2.8 \%$ in one study to $21.4 \%$ in another where orbital floor fractures were the second most common facial injury. Recognized sequelae of orbital floor blowout fractures include enophthalmos, diplopia from extraocular muscle dysfunction, orbital dystopia and infraorbital paresthesia. Special attention has to be paid to the posterior third of the orbit and the bony optic canal. Bony dislocations in these anatomical areas are more likely to be associated with traumatic optic nerve lesions.

A biomaterial is broadly defined as a natural or synthetic substance that is suitable to be incorporated into a living tissue particularly to aid healing, correct deformities and restore lost function. A perfect biomaterial would be chemically inert, non-allergenic and noncarcinogenic. It should also be cost effective to place, readily available and sterilizable, easy to handle, has the ability to be stable and retain its shape once manipulated. Preferably, it thought to be radiopaque to enable radiographic evaluation but without producing artifacts which may mask important features on subsequent radiological examination. 
Polypropylene is one of the most inert biomaterials used in surgery, and is available as a woven mesh (i.e., Prolene). It is easy to suture, has good tensile strength, and demonstrates early fibrous tissue in growth that serves to fix and incorporate the mesh.

Titanium is the most recently developed alloy. It is available as pure titanium, as well as a stronger alloy of titanium in combination with aluminum and vanadium. In the unalloyed form, titanium is more malleable than stainless steel or Vitallium, which facilitates easy and precise molding to fit the contours of the facial skeleton.

The purpose of this series was to assess the aesthetic and functional outcome of the orbital floor reconstruction performed with prolene mesh and that with titanium mesh.

\section{Material and Methods}

Thirty cases of orbital blow out fractures treated at Plastic Surgery Department Tirunelveli Medical College from January 2014toOctober 2019 were considered in this study. Clinical examination and radiological investigations were used.

Fifteen cases out of thirty were reconstructed by titanium mesh while the remaining fifteen cases were reconstructed by prolene mesh. Infraorbital approach is required for exposure of the orbital floor. Reconstruction of the orbital floor has to respect the course of the infraorbital nerve in the orbital floor. The forced duction test performed to determine ocular motility as soon as local anesthesia and IV sedation is induced. As soon as the infraorbital area has been dissected another forced duction test performed to determine ocular motility. After the insertion of any implant material, forced duction test to assure that the implant does not decrease the ocular motility. A careful assessment of the defect size should be performed preoperatively with the CT scan in the sagittal view which is in the course of the orbital nerve, plus the coronal view showing the transverse extent. It is crucial that in case of combined fracture types (e.g., displaced zygoma fracture) the final defect size is only measured after proper repositioning of the outer frame. The defect size can be measured by the reading on the orbital retractor or any other instrument. When using the fan-shaped plate, the outer circumference of the mesh is widest in the area of the infraorbital rim. The mesh should be trimmed so that the outer circumference is as small as possible but still provides enough width to cover the defect. Care has to be taken that neither orbital fat nor muscles are entrapped. If fixation is required, one screw will suffice in most cases. The screw can be placed into the floor of the orbit just posterior to the infraorbital rim. In case of prolene mesh 2 prolene sutures 4-0 fixed to periosteum of the infraorbital rim usually enough to fix the mesh in place.

\section{Results}

Prolene mesh was used in fifteen cases, titanium mesh was used in fifteen cases. All patients had significant improvement in their esthetic appearance. Symmetry was restored in all cases. All thirty cases had a noticeable improvement in the function. 10 cases out of thirty had diplopia preoperatively, all ten recovered completely following surgery. All cases with enophthalmos recovered completely after surgery. Nine patients with infraorbital numbness, all recovered completely during the six months following surgery. Non-preformed titanium mesh were used in fifteen patients for orbital floor repair. One patient did not state follow-up period but there was improvement in the outcome measures of diplopia and enophthalmos in this patient. In this study titanium mesh found to have good availability, stability, contouring, radiopacity, spaces within the mesh to allow dissipation of fluids and no donor site needed, so titanium mesh found to have excellent results with defects more than $2 \mathrm{~cm}$. The titanium was found to be expensive and possible sharp edges if not properly trimmed which may limit the ocular motility.

The prolene mesh found to have good availability, contouring, smooth edges and allows tissue in growth. While the disadvantages were, not radio- 
opaque (not visible on postoperative images), Lack of rigidity when a very thin mesh is used and so prolene mesh found to be used in all defects with better results.

\section{Discussion}

The study recommend that Polypropylene (prolene) meshes can be used for all bone defects for their pliability and their ability to deflect the weight of the orbit content when even up to three layers are overlapped. It has been employed to reconstruct part of the orbit in cases where bone matter has been lost. From the success obtained in other anatomical loci and with different functions, its utilization in reconstructing orbit bones is promising. The use of polypropylene mesh to rebuild orbits in the case of blow-out fractures has been reported in the literature.

Titanium is highly biocompatible, has a low infection rate with minimal resorption potential and well recognized osteo-integration. Large defects in the internal orbit have been reconstructed with titanium mesh with positive results as they are rigid enough to support the orbital contents, allowing better adaptation to individual anatomy. No significant differences in orbital volume restoration were noted using either technique.

The authors concluded that both techniques were successful and allowed for close reproduction of the natural orbital volume and shape; reduction of binocular single vision (BSV) loss area, correction of primary globe position and a significant improvement.

Titanium mesh had its drawbacks such as the risk of graft extrusion or anticipated difficulty of removing these materials if needed because of the fibrous in growth and the possibility of osseous overgrowth but none of the studies reviewed reported about these incidences.

\section{Conclusion}

Prolene mesh is recommended for all orbital floor defects with enopthalmos and restricted ocular movements, while in larger defects and impure blowout fractures involving the infraorbital rim, titanium mesh is recommended. The outcome of surgery with both materials was satisfactory. No postoperative complications were seen. Titanium mesh \& prolene mesh, has the potential to be useful reconstructive materials in orbital floor blowout fractures.

The prolene mesh found to have good availability, contouring, smooth edges and allows tissue in growth. While the disadvantages were, not radioopaque (not visible on postoperative images), lack of rigidity when a very thin mesh is used (one more mesh can be superimposed) and so prolene mesh found to be used in all defects with better results.

Titanium mesh had its drawbacks such as the risk of graft extrusion or anticipated difficulty of removing these materials if needed because of the fibrous in growth and the possibility of osseous overgrowth but none of the studies reviewed reported about these incidences.

\section{References}

1. Smith B. and Converse J.M.: Early treatment of orbital floor fractures. Trans. Am. Acad. Ophthalmol. Otolaryngol., 61: 602, 1957.

2. Fujino T.: Experimental blowout fracture of the orbit. Plast. Reconstr. Surg., 54: 81, 1974.

3. Erling B.F., Iliff N., Robertson B. and Manson P.N.: Footprints of the globe: A practical look at the mechanism of orbital blowout fractures, with a revisit to the work of Raymond Pfeiffer. Plast. Reconstr. Surg., 103: 1313, 1999.

4. Ellis E., El-Attar A. and Moos K.F.: An analysis of 2067 cases of zygomaticoorbital fracture. J. Oral Maxillofac. Surg., 43: 417, 1985.

5. Scherer M., Sullivan W.G., Jr. D.J.S., Phillips L.G. and Robson M.C.: An analysis of 1423 facial fractures in 788 patients at an urban trauma center. J. Trauma, 29, 1989. 
6. Burnstine M.A.: Clinical recommendations for repair of orbital facial fractures. Curr. Opin. Ophthalmol., 14: 236, 2003.

7. Hammer B.: Orbital Fractures: Diagnosis, Operative Treatment, Secondary Corrections. Kirkland, WA, Hogrefe \& Huber Publishers, p 100, 1995.

8. Park J. and Lakes R.S.; Biomaterials: An Introduction (ed3). New York, NY, Springer Science+Business Media, LLC, $\mathrm{p}$ 2, 2007.

9. Park J.B. and Bronzino J.D.: Biomaterials: Principles and applications. Boca Raton, FL, CRC Press LLC, p iv, 2003. 240 Vol. 40, No. 2 / Prolene or Titanium Mesh in Orbital Floor Reconstruction. 